\title{
Quench Simulation of REBCO Cable-In-Conduit Conductor with Twisted Stacked-Tape Cable
}

\author{
Rui Kang, Davide Uglietti, Rainer Wesche, Kamil Sedlak, Pierluigi Bruzzone, Yuntao Song
}

\begin{abstract}
Quench protection is a critical issue for magnet using high temperature superconducting (HTS) materials like REBCO. For cable-in-conduit conductor (CICC) with twisted REBCO tapes, the angular dependent critical current density $\left(J_{c}\right)$ of tape will further complicates the quench behavior. The CryoSoft code THEA is used to simulate the quench behavior of various sub-scale CICC in view of a quench experiment in SULTAN facility. The CICC are composed of the twisted tape stack assembled into copper shell (strand). It has been found that neglecting the angular dependence of $J_{c}$ in quench simulation will significantly underestimate the hot spot temperature therefore leave potential risk to damage the magnet. It is also shown that the contact thermal resistance between strand and the steel jacket plays a very important role on quench behavior. On the other hand, the interstrand electric/thermal resistance has very little influence.
\end{abstract}

Index Terms-High temperature superconductors, quench, cablein-conduit conductor, THEA, thermal-hydraulic

\section{INTRODUCTION}

$\mathrm{C}$ OATED conductor (or REBCO) tape is a candidate for high temperature superconducting magnets for future fusion devices. These magnets are designed either to work at higher temperature [1-4] and/or to achieve a higher magnetic field [3-5] above the range of $\mathrm{Nb}$ based low temperature superconductors (LTS). Thanks to the high critical temperature $\left(T_{c}\right)$, HTS materials are generally much more thermally stable than LTS. On the other hand, once a local quench happens in REBCO conductor, the excellent thermal stability will in turn slow down the quench propagation [6]. Consequently, temperature in the quenched region could reach extremely high value even before voltage exceeds the detection threshold. Therefore, despite HTS conductors are extraordinarily stable, study of quench protection is still crucial for a magnet employing HTS conductor taking into account the risk to damage the magnet.

The low normal zone propagation velocity (NZPV) of REBCO tape or coil has been studied by experiments and calculations [7-10]. However, for application in fusion magnet, tapes must be assembled in large conductors cooled by forced flow of helium. In such conductors quench behavior is more complex due to: (1) additional heat capacity of jacket and copper protection, (2) transverse current redistribution, and (3)

R. Kang is with the Department of Engineering and Applied Physics, University of Science and Technology of China, Hefei 230026, China. (E-mail: kangrui@mail.ustc.edu.cn). R. Kang is now a visiting student at EPFL Swiss Plasma Center, Ecublens 1015, Switzerland. heat transfer to forced flow supercritical helium. Accordingly, understanding quench development in large conductor is important for further optimization as well as design of quench protection system (QPS).

Some papers have reported on quench simulations in large HTS conductors. Despite of the different conductor layouts and operating condition, some general conclusions can be drawn. The thermal capacity of the steel jacket is beneficial to reduce the hot spot temperature $[2,11]$. The transverse heat propagation accelerated by reduced thermal resistance between tapes can also reduce the hot spot temperature [12]. However, in all these studies the angular dependent $J_{\mathrm{c}}$ of REBCO tape was neglected. $\mathrm{J}_{\mathrm{c}}$ of REBCO tape was always chosen as the value when field is parallel to the c-axis, which is the minimum. Note that at low temperature and high magnetic field, $J_{c}$ at field perpendicular to the c-axis can be five to six times higher than $J_{c}$ in the parallel field [13]. Usually cables should be fully or at least partially transposed to avoid current unbalance and twisting the tapes along longitudinal direction is often used to achieve transposition. The changing orientation between tape and magnetic field will then result into varying $\mathrm{J}_{\mathrm{c}}$. Although it may seem conservative to use the minimum $\mathrm{J}_{\mathrm{c}}$ for analysis, in the case of REBCO conductor this might eventually overestimate the quench propagation and underestimate the hot spot temperature.

At SPC, a REBCO CICC based on TSTC concept was designed for DEMO CS magnet [5, 14]. Quench experiments for a subscale conductor with three strands are now under preparation. In this paper, the sub-scale conductors are designed on one hand to retain all the essential properties of a full size conductor, and on the other hand to make possible the test in SULTAN. A model for conductors with multiple twisted strands was implemented in the CryoSoft code THEA. It has been used to simulate quench in several sub-scale conductors considering the test conditions in SULTAN. The influence of the contact thermal/electric resistance on quench behavior is studied. A homogeneous cable model with non-twisted tapes is also studied.

\section{MODELING OF THE SUB-SCALE CONDUCTOR}

\section{A. Scale Down the HTS Conductor for DEMO CS}

The full size HTS conductor for the CS magnet of EU DEMO

D. Uglietti, R. Wesche, K. Sedlak and P. Bruzzone are with the EPFL Swiss Plasma Center, Ecublens 1015, Switzerland (E-mail: davide.uglietti@psi.ch; rainer.wesche@psi.ch; kamil.sedlak@psi.ch; pierluigi.bruzzone@psi.ch).

Y.Song is with the Department of Institute of Plasma Physics, Chinese Academy of Sciences, Hefei 230031, China. (E-mail: songyt@ipp.ac.cn). 


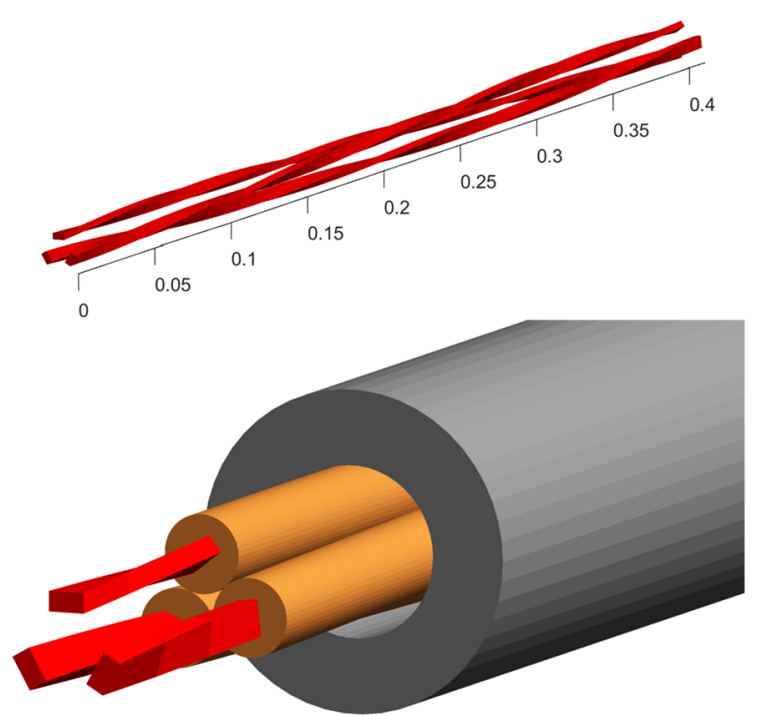

Fig. 1. Sketch of the sub-scale HTS conductor (bottom) and its twisted REBCO stacks (top) for the quench experiment. Strand diameter is $7 \mathrm{~mm}$ and each strand contains 15 tapes with width of $3.3 \mathrm{~mm}$.

is designed to carry $51 \mathrm{kA}$ current at $4.75 \mathrm{~K}$ and $17.5 \mathrm{~T}$; the current sharing temperature $\left(\mathrm{T}_{\mathrm{cs}}\right)$ is around $10 \mathrm{~K}$ [14]. During the test in the SULTAN facility, an $18 \mathrm{kA}, 10 \mathrm{~V}$ power supply will be used instead of the $100 \mathrm{kA}$ transformer, because the transformer is not able to provide current at voltages $>100 \mathrm{mV}$. Due to limitation of the sample current and maximum magnetic field in SULTAN (10.9 T), a sub-scale conductor that should have analogous behavior to the one of the full size (51 kA) is required for the quench experiment. The following strategies are used to scale down the full size conductor:

1) The operating current of the sub-scale conductor should be less than $18 \mathrm{kA}$.

2) The sub-scale conductor should have $T_{c s}$ around $10 \mathrm{~K}$ at peak field $10.9 \mathrm{~T}$.

3) The current density in copper should be about $100 \mathrm{~A} / \mathrm{mm}^{2}$. This is to ensure that the heat generation during quench is comparable to the situation in a full-size HTS cable, which also has current density around $100 \mathrm{~A} / \mathrm{mm}^{2}$.

4) Area of steel jacket is scaled down by the same ratio as area of copper.

5) The number of strands should be more than one to study transverse heat transfer and current sharing between strands.

Based on these strategies, a sub-scale conductor with three strands was envisaged, as shown in Fig. 1. Parameters of the full-size and sub-size conductors are compared in Table I. Calculation also shows that at $1 \mathrm{~g} / \mathrm{s}$ helium flow, the Reynolds numbers in the sub-scale and in the full size conductor are similar; therefore the cooling ability is also similar. In both fullscale and sub-scale conductor, the REBCO tapes are twisted in two stages. First, the stack of tapes is twisted in each strand and then the triplet is twisted together. In this work, the twist pitches for the two stages are respectively $0.4 \mathrm{~m}$ and $1.0 \mathrm{~m}$.

\section{B. Modeling of the Conductor}

For REBCO tape, the $\mathrm{J}_{\mathrm{c}}$ of the superconducting ceramic depends on temperature, magnetic field and the angle between
TABLE I

PARAMETERS OF FULL-SIZE AND SUB-SIZE CONDUCTOR

\begin{tabular}{lcc}
\hline \hline \multicolumn{1}{c}{ Parameter } & $\begin{array}{c}\text { Full-size } \\
\text { Conductor }\end{array}$ & $\begin{array}{c}\text { Sub-size } \\
\text { Conductor }\end{array}$ \\
\hline Operating temperature $(\mathrm{K})$ & 4.75 & 5 \\
Operating current $(\mathrm{kA})$ & 50.9 & 9.93 \\
Maximum magnetic field $(\mathrm{T})$ & 17.5 & 10.9 \\
Current sharing temperature $(\mathrm{K})$ & $\sim 10.5$ & $\sim 9.5$ \\
Copper area $\left(\mathrm{mm}^{2}\right)$ & 574 & 100 \\
Steel area $\left(\mathrm{mm}^{2}\right)$ & 2588 & 450 \\
Helium area $\left(\mathrm{mm}^{2}\right)$ & 151 & 64 \\
Total wetted perimeter $(\mathrm{mm})$ & 398 & 84 \\
Hydraulic diameter $(\mathrm{mm})$ & 1.52 & 2.93 \\
Operating pressure $($ bar) & 6 & 10 \\
Self-inductance of each $4.3 \mathrm{~m}$ strand $(\mu \mathrm{H})$ & & 2.69 \\
Mutual-inductance of each two strands $(\mu \mathrm{H})$ & & 1.87 \\
\hline \hline
\end{tabular}

TABLE II

SCALING PARAMETERS OF $\mathrm{J}_{\mathrm{C}}$

\begin{tabular}{lllcccc}
\hline \hline Birr0 $(\mathrm{T})$ & Tc $(\mathrm{K})$ & $\mathrm{A}(\mathrm{A} \cdot \mathrm{T} / \mathrm{m} 2)$ & $\alpha$ & $\beta$ & $\mathrm{p}$ & $\mathrm{q}$ \\
\hline 120 & 92.83 & $3.97 \times 10^{12}$ & 1.52 & 2.33 & 0.50 & 1.70 \\
\hline \hline
\end{tabular}

field and the c-axis of tape. The scaling relation for $J_{c}$ as function of temperature and field was updated for tapes from Shanghai Superconductor Technology Co., Ltd (they will be used for the full scale conductor) for field parallel to the caxis [5], as shown in equation (1-4). The new scaling parameters for the tape assumed in this study are summarized in Table II.

$$
\begin{gathered}
J_{c}(B, T)=\frac{A}{B}\left(\left(\frac{B_{i r r}(T)}{B_{\text {irr } 0}}\right)^{\beta}\right) b^{p}(1-b)^{q} \\
B_{i r r}(T)=B_{\text {irr } 0}(1-t)^{\alpha} \\
b=B / B_{\text {irr }}(T) \\
t=T / T_{c}
\end{gathered}
$$

In this study, the angular dependence of $J_{c}$ is modeled by Ginzburg-Landau (GL) scaling $\mathrm{J}_{\mathrm{c}}(\mathrm{B}, \theta)=\mathrm{J}_{\mathrm{c}}(\mathrm{Bf}(\theta))$, where $\theta$ is the angle between magnetic field and c-axis of tape and $\mathrm{f}(\theta)=\sqrt{\cos ^{2} \theta+\gamma^{-2} \sin ^{2} \theta}$. This scaling is however not accurate at high magnetic field [13]. Better fitting can be achieved if $\gamma$ is a function of temperature and magnetic field, instead of a constant. The best fitting $\gamma$ is calculated for each group of data (from $[13,15]$ ), as shown in Fig. 2. Then at any point of the B-T phase space, $\gamma(\mathrm{B}, \mathrm{T})$ is calculated by Lagrange interpolation. The magnetic field profile along the conductor in SULTAN, as well as the calculated strand $\mathrm{I}_{\mathrm{c}}$ are shown in Fig. 3.

The superconducting conductor is simulated with THEA code, which is based on a one-dimensional model. To avoid the boundary effect of helium for this short conductor, THEA simulation is interconnected with another code, FLOWER, which can simulate the whole helium circuit thus providing a credible boundary condition for THEA calculation. The coupling of these two codes is governed by code SuperMagnet. 


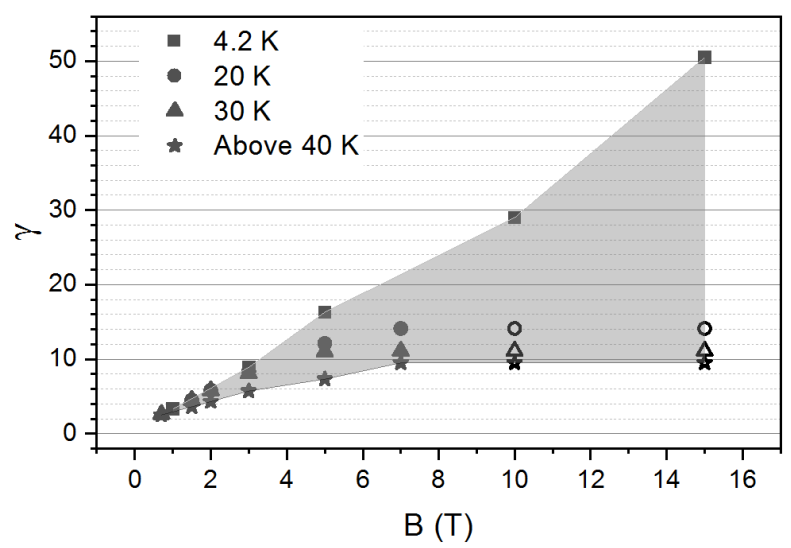

Fig. 2. Temperature and magnetic field dependence of $\gamma$. The solid points are derived from the experimental data. The open points are hypothetical values due to lack of experiment data at high temperature and high field. The gray region is the range of $\gamma$. The maximum field in SULTAN is $10.9 \mathrm{~T}$.

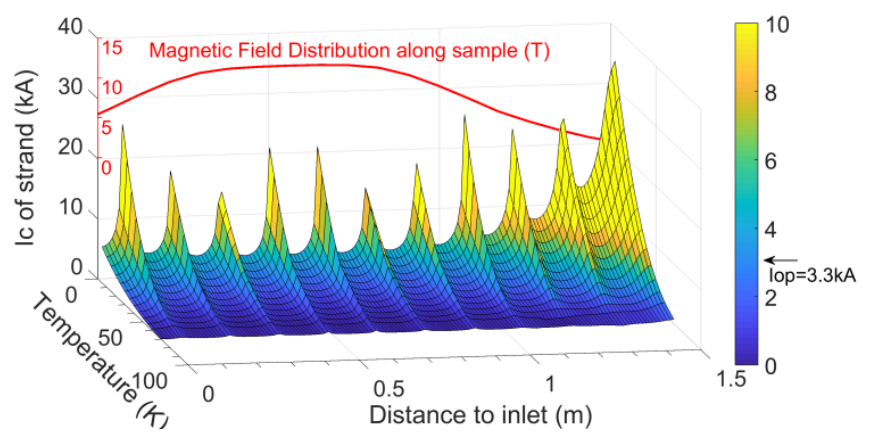

Fig. 3. Magnetic field and calculated critical current of strand No.1 (SN1) along the sub-scale conductor at different temperatures in SULTAN test conditions. Only first $1.5 \mathrm{~m}$ is shown, since beyond $1.5 \mathrm{~m}$ the magnetic field quickly reduces to little values. SN2 and SN3 show similar profile with a shift of the peaks.

Fig. 4 shows the helium circuit of this simulation. The physics model implemented in THEA and FLOWER as well as their coupling can be found in $[16,17]$. In the planned experiment the quench of the sub-scale conductor will be initiated by warm helium with temperature slightly above the $\mathrm{T}_{\mathrm{cs}}$ of the conductor at high field region; the same process is simulated here.

The sub-scale conductor modelled in THEA consists of five domains: one for each of the three strands, one for the steel jacket and one for the helium flowing through the voids. The strand $(\varnothing=7 \mathrm{~mm})$ consists of copper and REBCO tapes, and the thermal properties are considered homogeneous. Heat transfer and current sharing between strands are considered, but current sharing in steel jacket is set to zero. The c-axis of one strand is parallel to magnetic field at the position of maximum field (SN1 for strand No.1). The other two strands are rotated $120^{\circ}$ (SN2) and $240^{\circ}$ (SN3) with respect to SN1, as shown in Fig. 1. The inductance matrix is calculated by $\mathrm{M}^{\prime} \mathrm{C}$, CryoSoft [18].

In the first conductor model considered (BASIC), the interstrand electric resistance is set as $2 \mu \Omega \cdot \mathrm{m}$, which is the value measured by N. Bykovsky [11]. Following the assumptions from [19], the contact heat transfer coefficients between strandstrand and strand-jacket are both set at $500 \mathrm{~W} / \mathrm{m}^{2} \mathrm{~K}$. The contact length are both assumed 1/10 of the strand circumference. It follows that the contact thermal resistance is then $0.9 \mathrm{~K} \cdot \mathrm{m} / \mathrm{W}$.

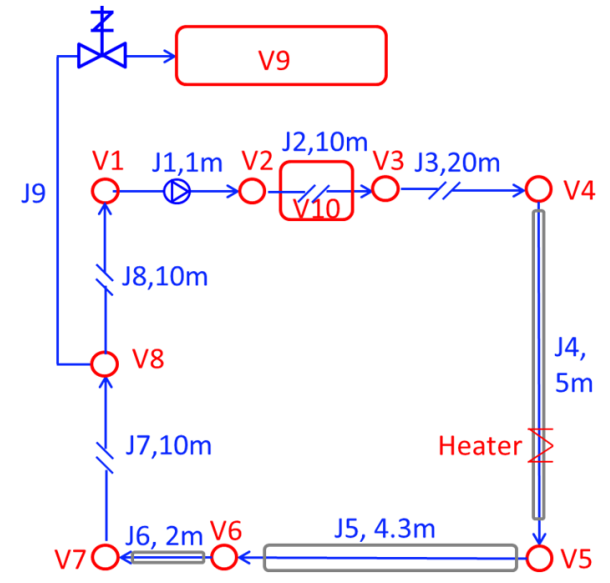

Fig. 4. Model of the helium circuit in Flower. The circuit includes a compressor J1 (1g/s), a re-cooling pipe J2 cooled by cold helium pool V10, connection pipe $\mathrm{J} 3, \mathrm{~J} 4, \mathrm{~J} 6, \mathrm{~J} 7$ and $\mathrm{J} 8$ and a pressure relief valve $\mathrm{J} 9$ as well as the relief pool V9. V1-V8 are only nodes between two pipes. J5 is the subscale conductor, which is simulated by THEA with helium boundary condition calculated by Flower.

TABLE III

CASES With DifFERENT CONTACT THERMaL/ELECTRIC RESISTANCE

\begin{tabular}{llll}
\hline \hline \multirow{2}{*}{$\begin{array}{c}\text { Conductor } \\
\text { model }\end{array}$} & $\begin{array}{c}\text { Inter-strand } \\
\text { electric resistance } \\
{[\mu \Omega \cdot \mathrm{m}]}\end{array}$ & \multicolumn{2}{c}{ Thermal resistance $[\mathrm{K} \cdot \mathrm{m} / \mathrm{W}]$} \\
\cline { 3 - 4 } & & Inter-strand & $\begin{array}{c}\text { Strand and } \\
\text { Jacket }\end{array}$ \\
\hline BASIC & 2 & 0.9 & 0.9 \\
LERS & 0.02 & 0.9 & 0.9 \\
HERS & 200 & 0.9 & 0.9 \\
LTRS & 2 & 0.009 & 0.9 \\
HTRS & 2 & 90 & 0.9 \\
LTR & 2 & 0.009 & 0.009 \\
HTR & 2 & 90 & 90 \\
NTH & $/$ & $/$ & 0.3 \\
\hline \hline
\end{tabular}

Clearly, the contact thermal resistance is the major uncertainty in this model. On the other hand, the contact thermal and electric resistance can both vary a lot for real conductors and they depend on temperature. Therefore, the effect of different contact thermal/electric resistances are also studied in the other conductor models (LERS to HTR), as shown in Table III: L or $\mathrm{H}$ stand for low or high, ER for electrical resistance, TR for thermal resistance, and $\mathrm{S}$ for strand. Although the initial motivation of such parametric study is to cover a wide range of the contact resistances to evaluate the sensitivity of $T_{\max }$ on the different resistances, the results later inspire some ideas to optimize conductor layout, as discussed in next section.

As comparison, one additional conductor model, named Non-Twisted Homogeneous conductor model (abbreviated as NTH) is considered. In NTH conductor model, the three strands are merged into one component with homogeneous thermal properties. Consequently, the inter-strand resistance is not meaningful anymore. In addition, the tapes are not twisted and the magnetic field is always parallel to the c-axis of the tapes. This is the model so far used in most of the quench simulation studies (see $[1,2,11,12,20]$ ). 


\section{RESULTS AND DISCUSSION}

\section{A. Quench Behavior of the Sub-Scale Conductor}

The planned quench experiment, and the simulations in this paper, can be divided into four phases: 1) quench initiation by warm helium (QI); 2) quench development (QD); 3) quench validation and QPS reaction (QV); and 4) current dump (CD).

Fig. 5 and Fig. 6 show the results of a complete quench simulation for the sub-scale conductor of the BASIC conductor model. Heater at J4 starts to produce warm helium at $0 \mathrm{~s}$. Then after $10 \mathrm{~s}$ warm helium arrives at the high field region and quench is initiated within $0.16 \mathrm{~m}$ initial length. The quench length is however not continuous along the sample due to the fluctuation of $T_{c s}$, which is result of the angular dependence of $\mathrm{J}_{\mathrm{c}}$. For the same reason, in the first few seconds when quench is developing, the temperature profile of strand shows fluctuation. However, soon with temperature increasing, the angular dependence of $\mathrm{J}_{\mathrm{c}}$ and the associated fluctuations of temperature profile decreases and finally disappear when $T_{c}$ is exceeded. Assuming $100 \mathrm{mV}$ quench detection voltage, quench is detected at $18.2 \mathrm{~s}$. After that, $1.1 \mathrm{~s}$ is assumed for validation of quench and activation of protection system. These two choices are also from [19]. The normal zone length reaches $0.84 \mathrm{~m}$ just before the exponential current dump at $19.3 \mathrm{~s}$. The average normal zone propagation velocity (NZPV) is thus about $75 \mathrm{~mm} / \mathrm{s}$. With the discharge time constant $\tau$ of $20 \mathrm{~s}$, which is a moderate value for CS magnet [21], maximum hot spot temperature $\left(\mathrm{T}_{\max }\right)$ in the strand will reach about $238 \mathrm{~K}$.

There is large temperature gradient among strand, helium and jacket at higher temperature. Such temperature gradient is also reported by R. Heller [20] and N. Bykovsky [11]. The large temperature gradient indicates that transverse heat transfer is relatively inefficient at higher temperature, especially between strand and jacket.

\section{B. Influence of Contact Thermal/Electric Resistance}

Fig. 7 summarizes the $T_{\max }$ and average NZPV of the conductor models presented in Table 3. As discussed above, in the BASIC conductor model, the thermal resistance between jacket and strands are relatively high and the huge heat capacity of the jacket is not exploited. If better heat transfer could be obtained (conductor model LTR in Fig. 7) $T_{\max }$ of strand can be significantly reduced, despite that NZPV is also reduced. It is worth mentioning that a large temperature reduction in the strands is obtained with a modest increase of temperature in the jacket, because of the large amount of steel and its large heat capacity at higher temperature. In view of the fact that in the CS magnet of DEMO reactor, a mass of steel is always indispensable for mechanical support, it would be beneficial to find a proper method to obtain low thermal resistance between the cable and jacket to reduce the $\mathrm{T}_{\max }$ of the HTS cable.

The inter-strand thermal/electric resistance surprisingly shows very little influence on $T_{\max }$ and NZPV, despite the fact that lower inter-strand resistance does have some interesting influence on the quench development, as shown in Fig. 8. Either excellent current redistribution or heat transfer can neutralize the effect of angular dependence of $\mathrm{J}_{\mathrm{c}}$ of each strand and

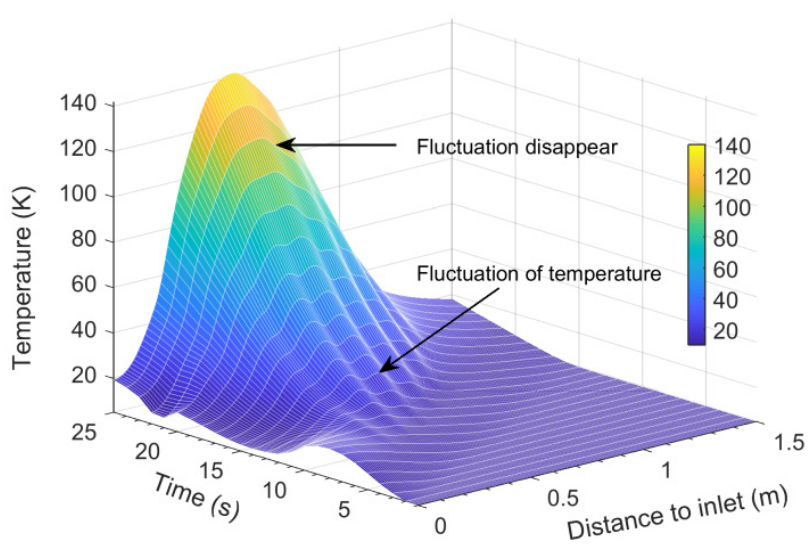

Fig. 5. Temperature profile of SN1 during first $25 \mathrm{~s}$ for the BASIC conductor model. Fluctuation of temperature appears at the beginning of quench development and then gradually disappears. SN2 and SN3 show similar distribution but with a shift of the fluctuation peaks and finally the same disappearance of fluctuations. As a result, SN1 will be used to present the result of the cable part in the following sections.
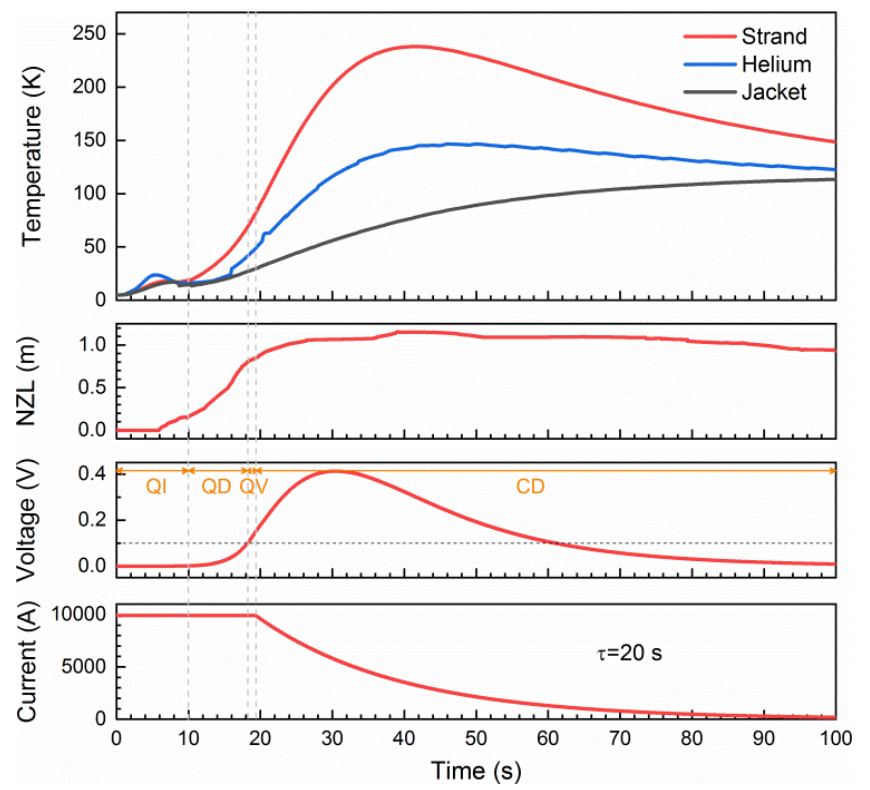

Fig. 6. Hot spot temperature and normal zone length (NZL) of SN1, and total resistive voltage and current of the BASIC conductor model as function of time in the four phases of quench simulation.

decrease the temperature during QD. However, the quench detection is also delayed, during which time the temperature continues to rise. Therefore, $\mathrm{T}_{\max }$ will finally reach values comparable to or even higher than the values in high resistance cases. In any case, this difference is too little to be attractive.

These results indicate that in term of quench protection, the inter-strand resistance is of little importance, while controlling the contact thermal resistance between cable and the jacket is crucial to control $\mathrm{T}_{\max }$. Few sub-scale conductors with different layouts will be prepared for the upcoming quench experiment to verify these results.

On the other hand, the contact resistances assumed in this paper does not dependent on temperature. Besides, the latest research shows that non-negligible temperature gradients are 


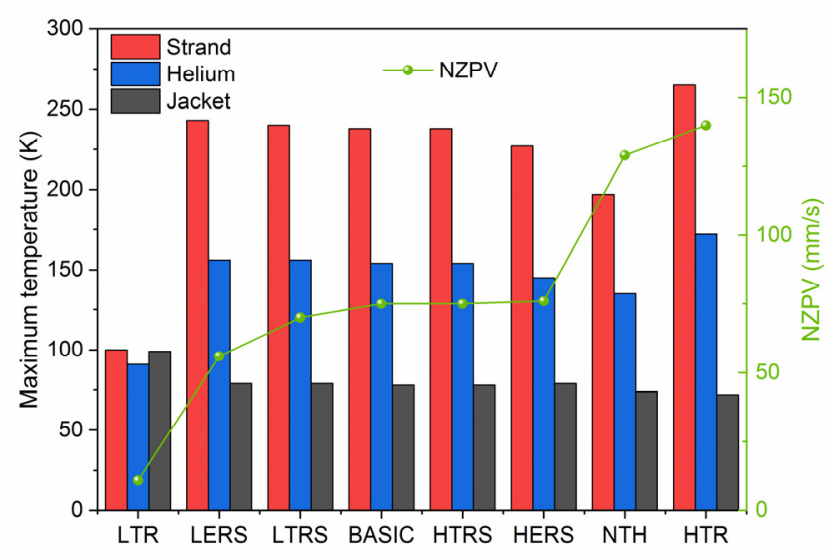

Fig. 7. Hot spot temperature and NZPV with different contact thermal/electric resistance.
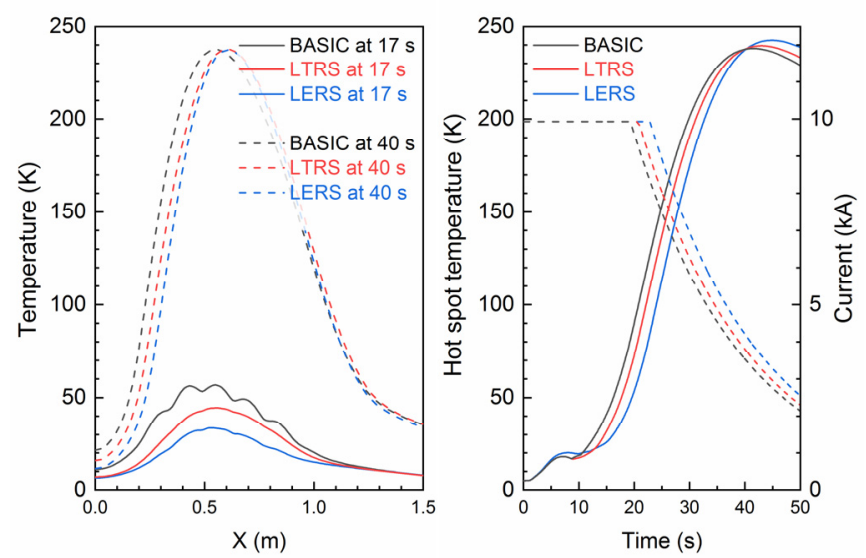

Fig. 8. Comparison of three cases with different inter-strand resistance. The left figure shows temperature profile of $\mathrm{SN} 1$ at two different times corresponding to $\mathrm{QD}$ and $\mathrm{CD}$ phases. The right figure shows $\mathrm{T}_{\max }$ (solid lines) and current (dash lines) as function of time. Fluctuation of temperature profile disappears with lower inter-strand thermal/electric resistance.

expected within one single strand, especially in a fast transient quench event [22]. Therefore, a more detailed simulation model may be needed.

\section{Result of Non-Twisted Homogeneous Model}

According to the results shown in Fig. 7, the NZPV calculated for the NTH conductor model is about twice faster and the $\mathrm{T}_{\max }$ is $40 \mathrm{~K}$ lower compared to the BASIC conductor model. Considering the little influence of the inter-strand resistance on the $T_{\max }$, this validates the hypothesis that using minimum $J_{c}$ and neglecting its angular field dependence is not really a conservative assumption for a quench analysis of REBCO conductor with twisted tapes and may lead to underestimating the maximum hot spot temperature.

\section{Discussion about the $T_{\max }$}

It is usually considered that the maximum temperature of the cable should not be higher than $200 \mathrm{~K}$ criterion [6]. All but one of the conductor models considered here (see fig. 7), have maximum temperatures close or higher than the limit, irrespective of the NZPV and of the presence of temperature fluctuations along the length.
There are several options to reduce hot spot temperature, like increasing the helium mass flow rate, reducing the detection threshold or decreasing the discharge time constant, but their implementations have consequences on the whole magnet system, and could be technically difficult or expensive.

One way to decrease hot spot temperature at the conductor design level is increasing the area of copper. An increase of $66 \%$ of the copper cross section would lower the maximum temperature to $160 \mathrm{~K}$ in the BASIC conductor model, but it will also decrease the total engineering current density and increase the total conductor space, eventually reducing the maximum field.

Instead, if good heat transfer between strands and jacket can be achieved (i.e. LTR), the maximum temperature is only 100 $\mathrm{K}$ and even if the copper is reduced by $33 \%$ the maximum temperature would be only few $\mathrm{K}$ higher. One possible way to have a rather low strand-jacket thermal resistance is to solder them together. Such conductor could have an acceptable maximum temperature with a cross section at least three times lower than in a cable not soldered to the jacket. As mentioned above, few sub-scale conductors with different layouts will be prepared for the upcoming quench experiment to verify these results.

\section{CONCLUSION}

A thermal-electrical-hydraulic model in CryoSoft THEA has been implemented to study the quench behavior of multi strands, twisted stacked conductors. The angular dependent $\mathrm{J}_{\mathrm{c}}$ in the REBCO tapes and the twisting of the cable are considered in this model as well as the transverse thermal/electric coupling among the strands.

During quench, the angular-dependent $J_{c}$ in the strands causes fluctuations in the temperature profile before quench temperature exceeds the $T_{\mathfrak{c}}$. The poor heat transfer between strand and jacket leads to a large temperature gradient between them.

The non-twisted homogeneous model neglecting angular dependence of $J_{c}$ and assuming enhanced thermal/electrical conductance between strands overestimates the NZPV and underestimates the quench hot spot temperature. Therefore, it is crucial to include the angular dependence of the HTS tapes for quench simulation of a conductor with twisted REBCO tapes.

Lower inter-strand resistance can neutralize the influence of angular dependence of $\mathrm{J}_{\mathfrak{c}}$, but has little influence on the hot spot temperature. Low thermal resistance between cable and jacket could strongly reduce the hot spot temperature, even though it also slows down the quench propagation. If the thermal resistance between cable and jacket is low, the amount of copper protection could be drastically reduced without affecting much the maximum temperature.

It is planned to verify these numerical predictions in experiments with different conductor layouts.

\section{ACKNOWLEDGEMENT}

Rui Kang gratefully acknowledges the financial support from China Scholarship Council. 


\section{REFERENCE}

[1] S. Hahn et al., "Design Study on a 100-kA/20-K HTS Cable for Fusion Magnets," IEEE Transactions on Applied Superconductivity, vol. 25, no. 3, pp. 1-5, 2015.

[2] N. Yanagi et al., "Magnet design with 100-kA HTS STARS conductors for the helical fusion reactor," Cryogenics, vol. 80, pp. 243-249, 2016.

[3] A. Sykes et al., "Compact fusion energy based on the spherical tokamak," Nuclear Fusion, vol. 58, no. 1, Jan 2018.

[4] D. G. Whyte, J. Minervini, B. LaBombard, E. Marmar, L. Bromberg, and M. Greenwald, "Smaller \& Sooner: Exploiting High Magnetic Fields from New Superconductors for a More Attractive Fusion Energy Development Path," Journal of Fusion Energy, vol. 35, no. 1, pp. 41-53, Feb 2016.

[5] R. Wesche et al., "Central solenoid winding pack design for DEMO," Fusion Engineering and Design, vol. 124, pp. 8285, Nov 2017.

[6] Y. Iwasa, Case Study in Superconducting Magnets. Springer US, 2009, pp. 469, 484-489.

[7] J. v. Nugteren, "Normal Zone Propagation in a YBCO Superconducting Tape," Master, University of Twente, 2012.

[8] Y. Shiroyanagi, W. B. Sampson, and A. K. Ghosh, "Quench propagation studies using a small bifilar YBCO coil," in Advances in Cryogenic Engineering, Vol 58, vol. 1435, U. Balachandran, Ed. (AIP Conference Proceedings, 2012, pp. 265-272.

[9] T. Lecrevisse et al., "Quench Propagation in YBCO Pancake: Experimental and Computational Results," IEEE Transactions on Applied Superconductivity, vol. 23, no. 3, Jun 2013, Art. no. 4601805.

[10] M. Bonura and C. Senatore, "An equation for the quench propagation velocity valid for high field magnet use of REBCO coated conductors," Applied Physics Letters, Article vol. 108, no. 24, p. 4, Jun 2016, Art. no. 242602.

[11] N. Bykovsky, "HTS high current cable for fusion application," PhD, EPFL, 2017.

[12] C. Marinucci, L. Bottura, M. Calvi, and R. Wesche, "Quench Analysis of a High-Current Forced-Flow HTS Conductor Model for Fusion Magnets," IEEE Transactions on Applied Superconductivity, Article vol. 21, no. 3, pp. 24452448, Jun 2011.

[13] A. Xu et al., "Angular dependence of Jcfor YBCO coated conductors at low temperature and very high magnetic fields," Superconductor Science and Technology, vol. 23, no. 1, p. 014003, 2010.

[14] R. Wesche et al., "DEMO Central Solenoid Design Based on the Use of HTS Sections at Highest Magnetic Field," IEEE Transactions on Applied Superconductivity, vol. 28, no. 3, Apr 2018.

[15] W. Stuart and S. Nick. Critical current characterisation of Shanghai Superconductor 2G HTS superconducting wire [Online]. Available: https://figshare.com/articles/Critical_current_characterisation of_Shanghai_Superconductor_2G_HTS_superconducting_wir e/5331145

[16] L. Bottura and C. Rosso, "Flower, a model for the analysis of hydraulic networks and processes," Cryogenics, vol. 43, no. 3-5, pp. 215-223, Mar-May 2003.
[17] L. Bottura, "Modelling stability in superconducting cables," Physica C, vol. 310, no. 1-4, pp. 316-326, Dec 1998.

[18] CryoSoft. Magnetic field, inductance, vector potential, energy, forces and $\mathrm{AC}$ loss analysis in a 3-D superconducting coil system of arbitrary shape [Online]. Available: https://supermagnet.sourceforge.io/manuals/Mac 2.9.pdf

[19] K. Sedlak, P. Bruzzone, and M. Lewandowska, "Thermalhydraulic and quench analysis of the DEMO toroidal field winding pack WP1," Fusion Engineering and Design, 2017.

[20] R. Heller, P. V. Gade, W. H. Fietz, T. Vogel, and K. P. Weiss, "Conceptual Design Improvement of a Toroidal Field Coil for EU DEMO Using High-Temperature Superconductors," IEEE Transactions on Applied Superconductivity, vol. 26, no. 4, Jun 2016.

[21] A. Torre, D. Ciazynski, and L. Zani, "EU-DEMO TF and CS Magnet Systems Design and Analyses Performed at CEA," Ieee Transactions on Applied Superconductivity, vol. 27, no. 4, Jun 2017.

[22] A. Zappatore, W. H. Fietz, R. Heller, L. Savoldi, M. J. Wolf, and R. Zanino, "A quasi-3D thermal-hydraulic model for an HTS CroCo conductor," presented at the 30th International Symposium on Fusion Technology (SOFT 2018), Giardini Naxos, I, September 16-21, 2018, 2018. 